Contors, D. E. (1959). J. gen. Microbiol. 21, 40-50

\title{
Kinetics of Bacterial Growth: Relationship between Population Density and Specific Growth Rate of Continuous Cultures
}

\author{
By D. E. CONTOIS* \\ Department of Marine Biology, University of California, La Jolla, California, U.S.A.
}

SUMMARY: Results from studies of continuous cultures of Aerobacter aerogenes growing in chemically defined media indicate that specific growth rate $(R)$ is a function of population density $(P)$ as well as the concentration of the limiting nutrient $(S)$. From these observations, and those of others, the following model for bacterial growth is derived:

$$
\boldsymbol{R}=\frac{u_{m} \boldsymbol{S}}{\boldsymbol{B P}+\boldsymbol{S}}
$$

where $u_{m}$ and $\boldsymbol{B}$ are growth parameters that are constants under defined conditions. This model is believed to have general applicability and to account for bacterial growth in both batch and continuous cultures.

Although biologists have long recognized that the specific growth rate of an organism is often a function of its population density (Pearl \& Parker, 1922; Greenleaf, 1926), most bacteriologists are of the opinion that the specific growth rate of a population of bacteria is, ideally, independent of population density. This idea has come down into recent treatments of bacterial growth kinetics of both batch and continuous cultures; and most workers have regarded the specific growth rate of a population growing under conditions of nutrient limitation as a function only of the concentration of the limiting nutrient (Monod, 1942, 1950; Novick \& Szilard, 1950; Spicer, 1955; Herbert, Elsworth \& Telling, 1956; Moser, 1958). Nevertheless, evidence supporting the contention that specific growth rates of bacterial populations are also functions of population density may be found in the old theories and concepts of 'cell space' (Bail, 1929). Contois (1957) presented evidence to this effect from studies of batch cultures growing under conditions of nutrient limitation. This paper is a report upon further studies undertaken to ascertain the effect of population density upon specific growth rates of bacterial populations, and as a further attempt to derive a more satisfactory model for bacterial growth.

\section{METHODS}

Organism. A strain of Aerobacter aerogenes was used which grew well in mineral salts media containing ammonium as a nitrogen source and glucose or succinate as a carbon source. In each batch of medium, either the nitrogen source or the carbon source was limiting, all other components being present in excess.

* Present address : Department of Bacteriology, University of Hawaii, Honolulu, Hawaii, U.S.A. 
Culture media. These were compounded from the following stock aqueous solutions:

Solution A, glucose $200 \mathrm{~mm}$.

Solution B, succinic acid 200 mм.; neutralized to $\mathrm{pH} 6.8$ with $\mathrm{KOH}$.

Solution C. $\mathrm{NH}_{4} \mathrm{H}_{2} \mathrm{PO}_{4} 250$ mM.; $\left(\mathrm{NH}_{4}\right)_{2} \mathrm{HPO}_{4} 250$ mM.

Solution D. $\mathrm{MgSO}_{4} \cdot 7 \mathrm{H}_{2} \mathrm{O} 80 \mathrm{~mm}$; $\mathrm{FeSO}_{4} .7 \mathrm{H}_{2} \mathrm{O} 3 \cdot 5 \mathrm{~mm}$.; $\mathrm{MnCl}_{2} \cdot 4 \mathrm{H}_{2} \mathrm{O}$ 5.0 mM.; $\mathrm{HCl} 1.0 \mathrm{~mm}$.

Solution E. $\mathrm{Na}_{2} \mathrm{HPO}_{4} 250 \mathrm{~mm}$.; $\mathrm{KH}_{2} \mathrm{PO}_{4} 250 \mathrm{~mm}$.

Solution F. Tetrasodium salt of ethylenediaminetetraacetic acid $25 \mathrm{~mm}$.

All media contained $10 \mathrm{ml}$. solution $\mathrm{D}$ and $5 \cdot 0 \mathrm{ml}$. solution $\mathrm{F} / 1$. The volumes of solutions $\mathbf{C}$ and $\mathbf{A}$ or $\mathbf{B}$ were changed from time to time to give desired concentrations of ammonium and the selected carbon source. Once the volume of solution $\mathrm{C}$ was determined for a given batch or medium, a volume of solution $\mathbf{E}$ was added that was sufficient to give a final total phosphate concentration of $\mathrm{M} / 30$.

Media were sterilized by passage through sterile molecular filters (Millipore type PH, available from Millipore Filter Corp., Watertown 72, Massachusetts, U.S.A.) and dispensed by the usual aseptic techniques. Before inoculation, the $\mathrm{pH}$ value of all media was $6 \cdot 8$.

Method of cultivation. A continuous culture device similar in theory and design to the one developed by Novick \& Szilard (1950) was used. The flow rate was adjusted by means of a stopcock which controlled the rate at which fresh medium dripped into the culture vessel. Flow rates were estimated by measuring the volumes of effluent accumulating over known time intervals as well as by drop counts of the inflowing medium.

Culture volume was constant for any given culture, but was different from time to time depending upon the particular culture vessel used. All culture volumes were between 30 and $45 \mathrm{ml}$.

Mixing and aeration of cultures was accomplished by vigorous sparging with sterile air at a rate of $c .1 \cdot 0 \mathrm{l}$. air $/ \mathrm{ml}$. culture volume/hr.; and the culture device was designed so that the inflowing medium was blown into the culture by the stream of sterile air. In addition, two sterile buoyant rubber disks c. $8 \mathrm{~mm}$. in diameter were placed in each culture vessel. By their continuous rapid movement throughout the culture, they scrubbed the walls of the culture vessel and eliminated wall-growth completely. They were also self-cleansing.

Measurement of population density. Population density was measured in units of optical density by a Beckman model DU spectrophotometer (available from Beckman Instruments, Inc., Fullerton, California, U.S.A.) with $1.0 \mathrm{~cm}$. Pyrex cuvettes and at $420 \mathrm{~m} \mu$. Comparisons of optical densities with direct counts showed that an optical density of 1.0 corresponded to a direct count of c. $5 \times 10^{8}$ organisms $/ \mathrm{ml}$. Experience showed that optical density was a linear function of population density within values of optical density of $0 \cdot 0-0 \cdot 6$. In practice, a sample of a culture was removed aseptically from the culture vessel and diluted until its optical density was less than 0.4 unit. The resultant reading was multiplied by the dilution factor to give the correct population density in units of optical density. 
Estimation of concentration of limiting nutrient. Previous experience (Contois, 1957) had shown that with this organism and these media the uptake of the limiting nutrient was a linear function of the increase in population density, or

$$
c\left(S_{0}-S\right)=\left(P-P_{0}\right)
$$

where $S_{0}$ is the initial concentration of the limiting nutrient, $P_{0}$ is the initial population density, and $c$ is a yield coefficient. Studies by Dagley \& Hinshelwood (1938), Lodge \& Hinshelwood (1939), Monod (1942), Herbert et al. (1956) and Lawrence (1957) have shown that this relationship is valid under appropriate conditions with various limiting nutrients serving as either carbon, nitrogen, phosphorus, or sulphur sources.

In continuous cultures, $S_{0}$ is the concentration of the limiting nutrient in the inflowing medium. Since $P_{0}$ is usually insignificant and is washed out of the culture in time anyway (see Spicer, 1955) equation (1) reduces to

$$
c S=\left(c S_{0}-P\right),
$$

where $c S_{0}$ is equivalent to the maximum population density that an organism can attain in a particular medium.

In this study, estimates of the concentration of the limiting nutrient $(S)$ were calculated by equation (2) from measurements of population density $(P)$, the maximum attainable population density $\left(c S_{0}\right)$, and the known values of $S_{0}$. In some of the cultures in which the nitrogen source was limiting, its concentration also was measured directly. This was done by removing a sample from the culture vessel and diluting it $1 / 10$ with distilled water. The mixture was filtered immediately through a molecular filter (Millipore type PH) and a sample of the filtrate diluted and nesslerized. After sufficient time, colour development was measured by a Beckman DU spectrophotometer in $10 \mathrm{~cm}$. Pyrex cuvettes at $476 \mathrm{~m} \mu$. Estimates of the concentration of ammonium were obtained by comparison with standards.

Estimation of specific growth rates. Monod (1950) and Novick \& Szilard (1950) showed that in continuous cultures the change in population density in time is equal to the rate at which the population grows minus the rate at which it is washed out of the culture vessel, or

$$
\frac{d P}{d t}=R P-D P
$$

where $R$ is the specific growth rate and $D$, dilution rate, is defined as the rate of flow of medium through the culture vessel per unit volume of culture (see Herbert et al. 1956).

In these experiments, it was desired to obtain estimates of specific growth rate and concentration of limiting nutrient when cultures were at equilibrium. Under such conditions, $d P / d t$ of equation (3) is zero and $R$ is equal to $D$. Estimates (in units of $\mathrm{hr} .^{-1}$ ) of specific growth rate, then, were made from measurements of flow rate and the known culture volumes. Replicate estimates were found to have a standard error of $c .2 \%$ of the mean of the estimates.

Other observations. Periodic measurements of the $\mathrm{pH}$ value of cultures were 
also made as an aid to assuring that any drastic changes in environmental conditions would be observed. In addition, samples of cultures were examined from time to time during each experiment for the purity of the bacterial species being grown. This was done by microscopic examinations of stained preparations and by plating samples into differential media.

\section{RESULTS}

After sterilization, the apparatus was assembled, connected to a reservoir of sterile medium, and placed in an incubator at $27^{\circ}$. The culture vessel was filled with medium and inoculated from a stock culture of Aerobacter aerogenes. After growth had proceeded for several hours, fresh medium was allowed to flow through the culture vessel at a constant rate. Determinations of dilution rate

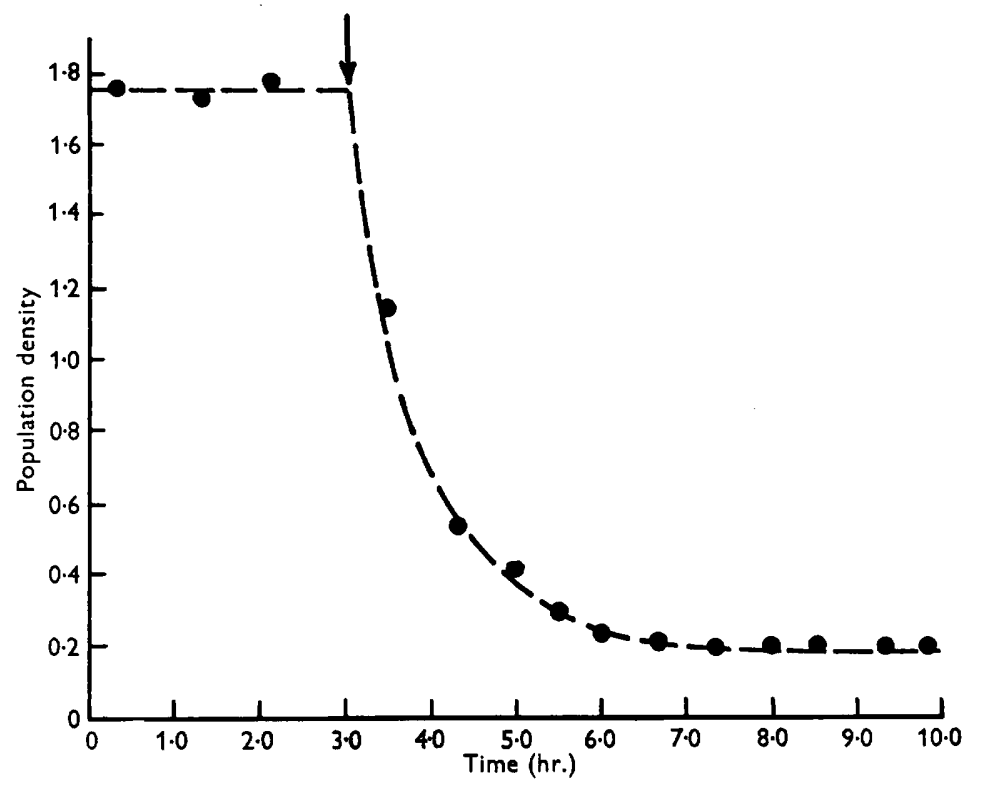

Fig. 1. Change in population density with change in dilution rate of a continuous culture of Aerobacter aerogenes with glucose limiting. Dilution rate changed from 0.50 to $1.05 \mathrm{hr}^{-1}$ at point indicated by arrow after culture had been in equilibrium for $c .17 \mathrm{hr}$.

(D) and population density were made periodically. After a time, the population density reached a constant value, indicating that equilibrium had been established. After several determinations of the equilibrium population density $\left(\boldsymbol{P}_{e}\right)$, the flow rate was adjusted to a new constant value and $\boldsymbol{P}_{e}$ was again determined. Two typical series of such measurements are shown in Fig. 1. By these methods, values of $\boldsymbol{R}$ and their corresponding values of $\boldsymbol{P}_{\boldsymbol{e}}$ were obtained for each culture (cf. Herbert et al. 1956).

Estimates of $c S_{0}$ were obtained by stopping the flow of medium and allowing the culture to attain its maximum population density at various times during each experiment. 
Table 1. Relationship between specific growth rate $(R)$, population density $\left(\boldsymbol{P}_{e}\right)$, and concentration of limiting nutrient $\left(S_{e}\right)$ in continuous cultures of Aerobacter aerogenes at equilibrium*

$$
\boldsymbol{R} \quad \boldsymbol{P}_{e} \quad\left(c S_{0}-P_{e}\right) \dagger \quad S_{e} \ddagger
$$

Culture $1 \mathrm{~A}$. Glucose as the carbon source and ammonium as the nitrogen source.

Glucose limiting at a concentration of $5.55 \mathrm{~mm}$. in the inflowing medium.

$\begin{array}{llll}1.07 & 0.49 & 2.03 & 4.48 \\ 0.97 & 1 \cdot 83 & 0.69 & 1.53 \\ 0.74 & 2 \cdot 20 & 0.32 & 0.71 \\ 0.49 & 2 \cdot 37 & 0.15 & 0.34 \\ 0.46 & 2 \cdot 40 & 0 \cdot 12 & 0 \cdot 25 \\ 0.00 & 2.52 & 0.00 & 0.00\end{array}$

Culture $1 \mathrm{~B}$. Glucose as the carbon source and ammonium as the nitrogen source. Ammonium limiting at a concentration of $3.75 \mathrm{~mm}$. in the inflowing medium.

\begin{tabular}{|c|c|c|c|}
\hline $1 \cdot 01$ & $0 \cdot 35$ & $1 \cdot 66$ & $3 \cdot 10$ \\
\hline $0 \cdot 83$ & 0.71 & $1 \cdot 30$ & $2 \cdot 42$ \\
\hline 0.79 & $1 \cdot 14$ & 0.87 & $1 \cdot 6$ \\
\hline $0 \cdot 44$ & 1.78 & $0 \cdot 23$ & 0.4 \\
\hline $0 \cdot 29$ & $1 \cdot 83$ & $0 \cdot 18$ & 0.3 \\
\hline 0.00 & 2.01 & 0.00 & \\
\hline
\end{tabular}

* Values of $R$ and $P_{e}$ are averages of several determinations.

$\dagger$ This term is an estimate of the concentration of the limiting nutrient in units of population density. The quantity $c S_{0}$ is constant for each culture and equal to the value of $P_{e}$ at $R$ equal to zero.

¥ Estimates of this term were calculated by means of equation (2) in the text and are expressed as millimoles per 1 .

Table 2. Relationship between specific growth rate $(R)$, population density $\left(\boldsymbol{P}_{e}\right)$, and concentration of limiting nutrient $\left(S_{e}\right)$ in continuous cultures of Aerobacter aerogenes at equilibrium*

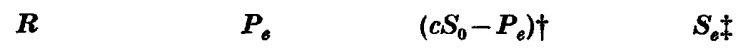

Culture 2A. Succinic acid as the carbon source and ammonium as the nitrogen source. Succinic acid limiting at a concentration of $8.47 \mathrm{~mm}$. in the inflowing medium.

$\begin{array}{llll}0.45 & 0.78 & 1.17 & 5.07 \\ 0.22 & 1.71 & 0.25 & 1.07 \\ 0.21 & 1.65 & 0.30 & 1.31 \\ 0.17 & 1.74 & 0.21 & 0.93 \\ 0.00 & 1.95 & 0.00 & 0.00\end{array}$

Culture 2B. Succinic acid as the carbon source and ammonium as the nitrogen source. Succinic acid limiting at a concentration of $16.95 \mathrm{~mm}$. in the inflowing medium.

$\begin{array}{rrrr}0.52 & 0.15 & 3.86 & 16.31 \\ 0.45 & 1.18 & 2.83 & 11.96 \\ 0.42 & 2 \cdot 17 & 1.84 & 7 \cdot 78 \\ 0.19 & \mathbf{3 . 6 3} & 0.38 & 1.61 \\ 0.00 & 4.01 & 0.00 & 0.00\end{array}$

* Values of $\boldsymbol{R}$ and $\boldsymbol{P}_{\mathrm{e}}$ are averages of several determinations.

$\dagger$ This term is an estimate of the concentration of the limiting nutrient in units of population density. The quantity $c S_{0}$ is constant for each culture and equal to the value of $P_{e}$ at $R$ equal to zero.

$\ddagger$ Estimates of this term were calculated by means of equation (2) in the text and are expressed as millimoles per 1 . 
Data from several experiments are given in Tables $\mathbf{1 - 3}$ and the relationships between $\boldsymbol{R}$ and $\boldsymbol{P}_{e}$ for each experiment are plotted in Fig. 2.

In cultures $3 \mathrm{~A}$ and $\mathbf{3 B}$ (data of Table 3 ), determinations of the concentration of the limiting nutrient (ammonium) were made by two independent

Table 3. Relationship between specific growth rate $(\boldsymbol{R})$, population density $\left(\boldsymbol{P}_{e}\right)$, and concentration of limiting nutrient $\left(S_{e}\right)$ in continuous cultures of Aerobacter aerogenes at equilibrium*

$$
R \quad P_{e} \quad\left(c S_{0}-P_{e}\right) \dagger \quad S_{e} \ddagger
$$

Culture 3A. Succinate as the carbon source and ammonium as the nitrogen source. Ammonium limiting at a concentration of 3.75 mm. in the inflowing medium.

$\begin{array}{llll}0.54 & 0.37 & 1.77 & - \\ 0.49 & 0.77 & 1.36 & 2.48 \\ 0.45 & 1.35 & 0.78 & 1.48 \\ 0.42 & 1.63 & 0.50 & 0.90 \\ 0.27 & 1.98 & 0.15 & 0.26 \\ 0.00 & 2.13 & 0.00 & 0.00\end{array}$

Culture 3B. Succinate as the carbon source and ammonium as the nitrogen source. Ammonium limiting at a concentration of $7.50 \mathrm{~mm}$. in the inflowing medium.

\begin{tabular}{|c|c|c|}
\hline 0.54 & $0 \cdot 20$ & 3.76 \\
\hline 0.50 & 1.43 & $2 \cdot 53$ \\
\hline $0 \cdot 44$ & $2 \cdot 36$ & $1 \cdot 60$ \\
\hline $0 \cdot 37$ & $\mathbf{3 \cdot 2 6}$ & $0 \cdot 70$ \\
\hline $0 \cdot 26$ & $3 \cdot 59$ & $0 \cdot 37$ \\
\hline 0.17 & $3 \cdot 59$ & 0.37 \\
\hline 0.00 & 3.96 & 0.00 \\
\hline
\end{tabular}

* Values of $R, P_{e}$, and $S_{e}$ are averages of several determinations.

$\dagger$ This term is an estimate of the concentration of the limiting nutrient in units of population density. The quantity $c S_{0}$ is constant for each culture and equal to value of $\boldsymbol{P}_{e}$ at $\boldsymbol{R}$ equal to zero.

$\ddagger$ Estimates of this term were made by direct chemical analysis and are expressed in millimoles per 1 .

methods: (a) by means of equation (2) from estimates of $P_{e}$ and $c S_{0}$; and (b) direct measurement of the concentration of ammonium. Figure 3 shows plots of estimates made by these two methods. The results confirm the relationship defined by equation (2) and show that the yield coefficient (the slope of the line in Fig. 3) is independent of specific growth rate.

Monod (1942) showed that the relationship between the specific growth rate of a bacterial population $(R)$ and the concentration of a limiting nutrient $(S)$ is approximately represented by

$$
\boldsymbol{R}=\frac{\boldsymbol{R}_{m} \boldsymbol{S}}{\boldsymbol{A}+\boldsymbol{S}}
$$

Parameters $\boldsymbol{R}_{m}$ and $A$ are, respectively, the maximum specific growth rate of the culture and the concentration of the limiting nutrient when the specific growth rate is $\frac{1}{2} R_{m}$. The same relationship was found by Schaefer (1948) and by Longmuir (1954).

At equilibrium in continuous cultures, from equations (3) and (4),

$$
D=R=\frac{R_{m} S_{e}}{A+S_{e}}
$$


where $S_{\varepsilon}$ is the concentration of limiting nutrient at equilibrium (Monod, 1950; Herbert et al. 1956; Moser, 1958). It is apparent from equation (5) that as $S_{e}$ approaches $S_{0}$ and, from equation (2), as $P_{e}$ approaches zero, $R$ approaches a critical value $R_{c}$ (see Herbert et al. 1956), where

$$
R_{c}=\frac{R_{m} S_{0}}{A+S_{0}}
$$

Estimates of $R_{c}$ can be obtained for each culture from the intersection on the $y$-axis of the curve giving $R$ as a function of $P_{e}$ (Fig. 2). Of particular interest is the fact that the estimates of $R_{c}$ are the same for each culture with the same nitrogen and carbon sources, irrespective of the kind or concentration of the limiting nutrient. Thus, from equation (6), the ratio of the values of $A$ for each of two such cultures is equal to the ratio of their values of $S_{0}$, or

$$
\frac{A^{\prime}}{A^{\prime \prime}}=\frac{S_{0}^{\prime}}{S_{0}^{\prime \prime}}
$$

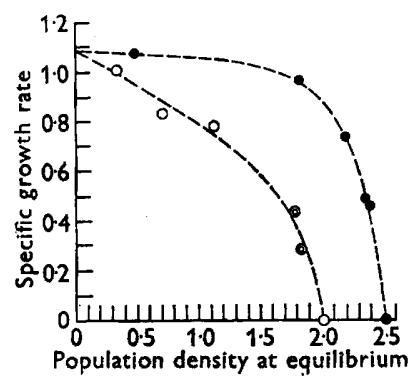

Fig. $2 a$

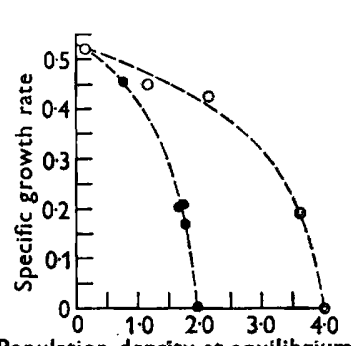

Fig. $2 b$

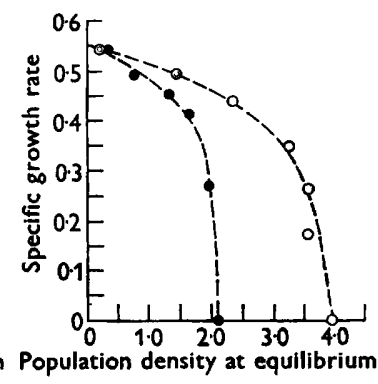

Fig. $2 c$

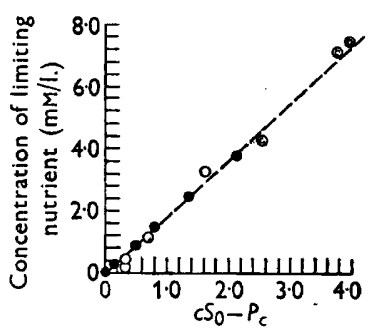

Fig. 3

Fig. $2 a, b$ and $c$. Relationship between specific growth rate and population density of continuous cultures of Aerobacter aerogenes at equilibrium. (a) Data of Table 1; 0 = culture $1 \mathrm{~A}$, glucose limiting at $5.55 \mathrm{~mm}$. in inflowing medium; $\mathrm{O}=$ culture $1 \mathrm{~B}$, ammonium limiting at $3.75 \mathrm{~mm}$. in inflowing medium, glucose as carbon source. (b) Data of Table 2; $=$ culture 2A, succinate limiting at $8.47 \mathrm{~mm}$. in inflowing medium; $\mathrm{O}=$ culture $2 \mathrm{~B}$, succinate limiting at $16.95 \mathrm{~mm}$. in inflowing medium. $(c)$ Data of Table 3; $\boldsymbol{Q}=$ culture $3 \mathrm{~A}$, ammonium limiting at $3.75 \mathrm{~mm}$. in inflowing medium, succinate as carbon source; $O=$ culture $3 \mathrm{~B}$, ammonium limiting at $7.50 \mathrm{mM}$. in inflowing medium, succinate as carbon source.

Fig. 3. Relationship between concentration of limiting nutrient (ammonium) and estimates of concentration of limiting nutrient in units of population density $\left(c S_{0}-P_{e}\right)$ in continuous cultures of Aerobacter aerogenes at equilibrium. Data of Table 3; $=$ culture 3A, $O=$ culture $3 \mathrm{~B}$. See text for definitions of terms. 
Since $S_{0}$ can be set at will, $A$ must be a function of $S_{0}$.

Further evidence of this relationship is obtained from consideration of the data of Tables 2 and 3. Rearrangement of equation (5) gives

$$
\frac{1}{R}=\frac{1}{R_{m}}+\left(\frac{A}{R_{m}}\right)\left(\frac{1}{S_{e}}\right)
$$

If $A$ is a function of $S_{0}, 1 / R$ should be a linear function of $1 / S_{e}$ for each culture, but with slope dependent upon $S_{0}$. Figure 4 shows regressions of $1 / S_{e}$ upon $1 / R$ for the data of Tables 2 and 3 fitted by the method of least squares. The slopes of the regression lines are, respectively, $3 \cdot 66$ and 5.90 for cultures $2 \mathrm{~A}$ and $2 \mathrm{~B}$;

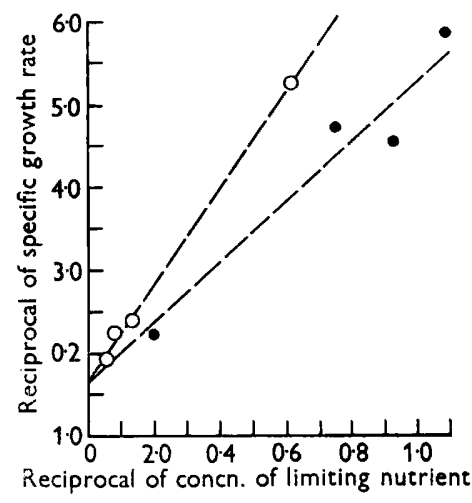

$\boldsymbol{a}$

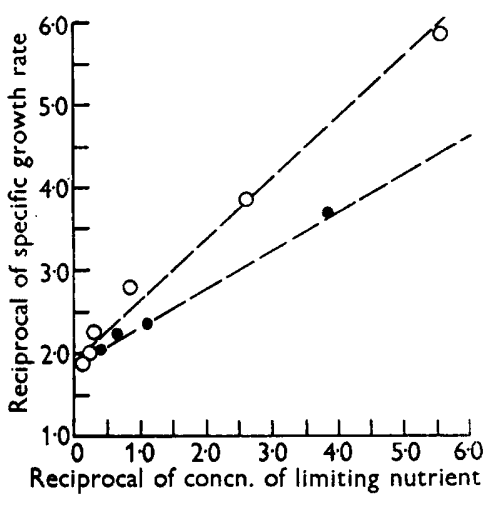

$b$

Fig. $4 a$ and $b$. Regression of reciprocals of the concentration of limiting nutrient upon reciprocals of specific growth rate in continuous cultures of Aerobacter aerogenes. (a) Data of Table 2; $=$ culture 2A, $\mathrm{O}=$ culture 2B. (b) Data of Table 3; $\mathrm{O}=$ culture $\mathbf{3 A}, \mathrm{O}=$ culture $\mathbf{3} \mathrm{B}$.

and 0.47 and 0.72 for cultures $3 \mathrm{~A}$ and $3 \mathrm{~B}$. These results show that the values of A are correlated with $S_{0}$ in each instance and can be considered to be proportional to $S_{0}$, or

$$
A=b S_{0} \text {. }
$$

Dr A. Novick (personal communication) has pointed out that $A=a+b^{\prime} S_{0}$ is a more realistic relationship in that on conversion of $A$ into terms of $P_{e}$ and $S_{e}$ by equation (2) and substitution into equation (4) a model is obtained that is better behaved at small values of $\boldsymbol{P}_{\boldsymbol{e}}$. However, the precision of the data is not good enough to determine whether or not $a$ is real. Moreover, equation (8) is sufficient for the purposes of this discussion since $a$, even if real, would be quite small compared to the values of $b S_{0}$ in these experiments.

Substituting equation (8) into (7) gives, therefore,

$$
\frac{1}{\boldsymbol{R}}=\frac{1}{R_{m}}+\left(\frac{b S_{0}}{R_{m}}\right)\left(\frac{1}{S_{e}}\right)
$$

and, from equation (2) and letting $(1+b) / R_{m}=1 / u_{m}$ and $b / c R_{m}=B / u_{m}$,

$$
\frac{\mathbf{1}}{\boldsymbol{R}}=\frac{\mathbf{1}}{u_{m}}+\left(\frac{\boldsymbol{B}}{u_{m}}\right)\left(\frac{\boldsymbol{P}_{e}}{S_{e}}\right)
$$


Equation (10) requires that the reciprocal of the specific growth rate should be a linear function of the ratio of population density to concentration of limiting nutrient. Plots of $1 / R$ versus $\boldsymbol{P}_{e} / S_{e}$ for the data of Tables $1-3$ are given in Fig. 5, and confirm the relationship given by equation (10). The different slopes of Fig. $5 a$ arise from the fact that the limiting nutrient is different in each culture.

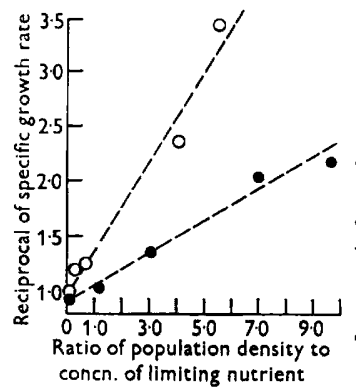

$a$

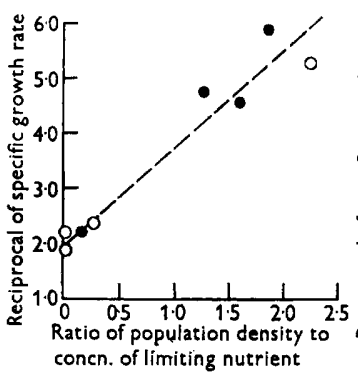

$b$

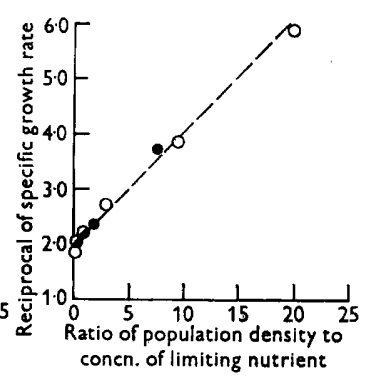

$c$

Fig. $5 a, b$ and $c$. Relationship between reciprocals of specific growth rate and ratios of population density to concentration of limiting nutrient in continuous cultures of Aerobacter aerogenes at equilibrium. (a) Data of Table 1 ; $=$ culture $1 \mathrm{~A}, \mathrm{O}=$ culture $1 \mathrm{~B}$. (b) Data of Table 2; $=$ culture $2 \mathrm{~A}, \mathrm{O}=$ culture $2 \mathrm{~B}$. (c) Data of Table 3 ; $\mathrm{O}=$ culture $3 \mathrm{~A}, \mathrm{O}=$ culture $\mathrm{3B}$. $\odot$ indicates two points at the same locus.

Rearrangement of equation (9) gives

and equation (10) gives

$$
R=\frac{R_{m} S}{b S_{0}+S}
$$

$$
R=\frac{u_{m} S}{B P+S}
$$

\section{DISCUSSION}

Monod (1942), in deriving equation (4), and others, who subsequently used this relationship, considered parameter $A$ to be independent of population density. Most of Monod's data, however, was obtained from experiments in which specific growth rates were measured at the beginning of growth cycles of batch cultures with different concentrations of limiting nutrient and in which initial population densities were the same from culture to culture. His results could be explained by equation (12) since, with population density essentially constant, equation (12) would reduce to equation (4). In studies of batch cultures where specific growth rates and concentrations of limiting nutrient were measured throughout the entire growth cycle, the relationship between specific growth rate and nutrient concentration for each culture could be expressed by equation (11); since, with initial concentration of limiting nutrient constant in each instance, equation (11) also reduces to equation (4).

Although the results of this study show that parameter A-the concentra- 
tion of the limiting nutrient when the specific growth rate is half maximumis a function of population density, the data provide no insight into the origin of or reason for this relationship. Other workers have attributed deviations of continuous cultures from Monod's model (equation (4)) to 'apparatus effects' such as incomplete or 'imperfect' mixing of the inflowing medium with the culture and wall-growth (Herbert et al. 1956). Although 'perfect' mixing is difficult to attain in continuous culture devices with large culture volumes, the small culture volumes $(30-45 \mathrm{ml}$.) and the methods of mixing and introduction of medium used in these studies would preclude incomplete mixing as a factor responsible for the observed results. Further tests, which consisted of introducing drops of a dye solution into the culture vessels, showed that mixing was completed in only 2 or $3 \mathrm{sec}$. As previously mentioned, no wall-growth was observed in any of these experiments.

The point could be legitimately raised that the observed relationship between population density and parameter $\mathbf{A}$ might be due to consequential changes in environmental conditions as population density becomes large, or to the possibility that oxygen may become limiting at high growth rates (cf. Pirt, 1957). The author believes that these factors were not operative in these studies since the observed growth parameters for each culture were independent of population density, nutrient concentration and specific growth rate. Also, under comparable conditions they were reproducible from culture to culture independently of the concentration of the limiting nutrient in the inflowing medium. The results observed in these experiments might be attributable to an inhibition of the growth process by end-products of that process. Since the yield coefficient is independent of specific growth rate in each culture, the concentrations of soluble end-products of the growth process probably are related to $\boldsymbol{P}$ (or $S_{0}$ ) in a simple manner. Thus the appearance of $\boldsymbol{P}$ in equation (12) may be due to what is primarily an effect of concentration of end-products.

The nature of equation (11) (or (12)) is such that, discounting lag, so-called logarithmic growth could never occur in batch cultures. However, parameter $b$ is small for most cultures; and, as a result, with population density small and concentration of limiting nutrient relatively large (conditions usually extant in 'young' batch cultures) growth could be considered logarithmic for all practical purposes over a considerable time interval. For example, an organism growing in batch culture with an initial population density of $1 \times 10^{6}$ organisms $/ \mathrm{ml}$., $c S_{0}$ equal to $2 \times 10^{9}$ organisms $/ \mathrm{ml}$., $R_{m}$ equal to $0.5 \mathrm{hr} .^{-1}$, and $b$ equal to $0 \cdot 1$ would have an initial specific growth rate (by equation (11)) of 0.454 . After c. $10 \mathrm{hr}$. of growth and at a population density of $1 \times 10^{8}$ organisms/ml. the specific growth rate would still be $\mathbf{0 . 4 5 2}$.

In conclusion, it is believed that equations (11) and (12) serve as adequate models for bacterial growth in both batch and continuous cultures and are more realistic than other models proposed previously. By substitution of equation (12) into equation (3) and integrating with dilution rate constant, a relationship is obtained which shows that population density and concentration of limiting nutrient approach constant values in time which are unique 
for each dilution rate less than the maximum specific growth rate. This accounts for the relationship between specific growth rate and population density at equilibrium that has been observed in this and other studies (see Herbert et al. 1956) and the fact that equilibrium is even attained by continuous cultures.

The author wishes to express his thanks and appreciation for the interest, encouragement and helpful suggestions given by Drs Claude ZoBell and Carl Eckart of the University of California, La Jolla; and the financial aid granted by the Steering Committee of the Marine Life Research Program of the University of California.

Contribution from the Scripps Institution of Oceanography, New Series.

\section{REFERENCES}

BaIL, O. (1929). Ergebnisse experimenteller Populationsforschung. Z. ImmunForsch. 60, 1.

Contors, D. E. (1957). Kinetics of Microbial Growth. Theory and Applications. Ph.D. thesis, University of California, Los Angeles.

Dagley, S. \& Hinshelwood, C. N. (1938). Physicochemical aspects of bacterial growth. I. Dependence of growth of Bact. lactis aerogenes on concentration of medium. J. chem. Soc. p. 1930.

Greenleaf, W. E. (1926). The influence of volume of culture medium and cell proximity on the rate of reproduction of infusoria. J. exp. Zool. 46, 143.

Herbert, D., Elsworth, R. \& Telling, R. C. (1956). The continuous culture of bacteria: a theoretical and experimental study. J. gen. Microbiol. 14, 601.

Lawnence, J. V. (1957). Bacterial growth and food supply. Bact. Proc. 57, 63.

Lodge, R. M. \& Hinsheuwood, C. N. (1939). Physicochemical aspects of bacterial growth. IV. Considerations determining stationary populations and growth rates of Bact. lactis aerogenes in synthetic media. J. chem. Soc. p. 1683.

Longmuir, I. S. (1954). Respiration rate of bacteria as a function of oxygen concentration. Biochem. J. 57, 81.

Monon, J. (1942). Recherches sur la Croissance des Cultures Bactériennes. Paris: Hermann et Cie.

Monod, J. (1950). La technique de culture continué. Théorie et applications. Ann. Inst. Pasteur, 79, 390.

Moser, H. (1958). The dynamics of bacterial populations maintained in the chemostat. Publ. Carneg. Instn, no. 614.

Novick, A. \& Szilard, L. (1950). Description of the chemostat. Science, 112, 715.

Pearl, R. \& Parker, S. L. (1922). On the influence of density of population upon the rate of reproduction in Drosophila. Proc. nat. Acad. Sci., Wash. 8, 212.

PIrT, J. (1957). The oxygen requirement of growing cultures of an Aerobacter species determined by means of the continuous culture technique. J. gen. Microbiol. 16, 59.

Schaefer, W. (1948). Recherches sur la croissance du Mycobacterium tuberculosis en culture homogené. Ann. Inst. Pasteur, 74, 458.

SPICER, C. C. (1955). The theory of bacterial constant growth apparatus. Biometrics, 11, 225.

(Received 30 September 1958) 